\title{
A Panoramic View of Fiber and Integrated Optics in Spain
}

\author{
J. A. MARTIN-PEREDA \\ E.T.S. Ing. Telecomunicación \\ Universidad Politécnica de Madrid \\ Ciudad Universitaria \\ 28040 Madrid, Spain \\ C. GOMEZ-REINO \\ Laboratorio de Optica \\ Departamento de Física Aplicada \\ Universidad de Santiago de Compostela \\ 15706 Santiago de Compostela, Spain
}

\begin{abstract}
A general view of the present status of optics and related fields in Spain is presented. The main emphasis is on the relation between optics and some emerging areas such as Optical Communications and Nonlinear Optics. Principal activities of the more important groups are summarized.
\end{abstract}

\section{Introduction}

Optics, Optoelectronics, Fiber Optics, Photonics, ... have had a very strange and, sometimes, difficult life in Spain. Almost none of the above fields have maintained an easy place among the different areas of Science and Technology, both in academic or industrial sectors. There have been many false starts, with strong efforts, several times. But after a period of time, sometimes long, sometimes short, these efforts petered out. The adventure of Optics and related fields was always initiated, almost in every case, just by personal initiatives. It was not the result, as it has been in many other countries, of some higher decision level.

As a matter of fact, these difficulties are not typical characteristics of just Optics, Optoelectronics, Fiber Optics, or Photonics. They have been present in almost every field of Science and Technology in Spain. Several studies have appeared in the last years discussing this problem and trying to determine the source of its roots. It is not the purpose of these pages to present some of the conclusions. But it is worthy to say that, in many cases, these problems were due to lack of coordination between the different agents involved in Science and Technology ( $\&$ \& $\mathrm{T}$ ) and the main responsibilities of Research and Development (R \& D). The Spanish system of S\&T has had no structure until recent years. Public authorities were not given, until the last decade, an instrument to coordinate different actions having similar objectives. Many of these actions could be stated at different ministerial departments. And no coordination among their $\mathrm{R} \& \mathrm{D}$ programs was existing. 
As will be shown in this article, the future may be better, because some initiatives have been arranged in the last years. Some of them are coming from the Spanish Government; some others from the Local Autonomous Governments; and some others, finally, from some industries operating in the Communications Sector. But the work of individuals is still the most powerful arm to advance the abovementioned fields in Spain.

The main objective of this paper is to present a realistic approach to the present situation of Spain in fields related to Fiber and Integrated Optics. Some history will be given at the beginning, because we think history is a way to gain a better understanding of why some areas are nowadays present in Spain and some others are still missing. It is obvious that this historical approach will be biased by the personal view of the authors. An objective history of Optics in Spain is still waiting for a writer. In any case, as will be shown, today appears brighter than yesterday, and the light is starting to shine over the Spanish panorama. A global view, with some statistical data, will be the second part of the paper. The third, and final one, will present a close approach to the activities of the main groups in the area. Not many details will be given in some of them, because their present research topics are more explicit in the papers that have been written for this special issue.

\section{Some History About Optics and Photonics in Spain}

Classical Optics has had a long tradition among the activities of the Spanish University. Two main centers have been present since the end of the Civil War, in 1939: Madrid and Zaragoza. It is easy to assert that many of the present professors and researchers in this area, mainly in nonengineering departments, are coming from the schools created by the two scientists who were in the two corresponding professorships, namely, Professors A. Duran and J. Casas. Moreover, at that time, an institute was created in Madrid, at the Higher Council for Scientific Research (CSIC). Its name is "Daza de Valdés Institute," and it had the aim of stimulating $\mathrm{R} \& \mathrm{D}$ in Optics in this institution.

On the other side, optical activities in engineering departments were almost nonexistent until the mid-sixties. In 1965 the first ruby laser was constructed, at the Telecommunication Engineering Department, of the Madrid Politechnical University (UPM), by some students under the direction of Professor A. Luque. This was the beginning of a group of engineers interested in the field of the new Optics. One of the authors of this paper was among those students. Professor Luque is working today in Photovoltaic Solar Energy, but he still has some interest in Optics, mainly in the area of Solar Concentrators.

Some activities in Modern Optics were initiated during the seventies, in several parts of Spain. Holography was initiated at Alicante, Madrid Complutense (UCM) and Madrid Politechnical Universities; several lines of Optical Communications at UPM and Politecnica de Cataluña (UPC); and studies on GaAs at UPM and Madrid Autonomous University (UAM).

Most of the resources were coming from the Advisory Commission for Science and Technology (CAYCIT), an special Agency at the Ministry of Education. But no priority lines were fixed. A horizontal program for Basic Research, with some tendency towards applied topics, was the only frame. This situation remained static until 1986. A new Law for Science and Technology was approved that year by the Spanish Parliament, and, with it, a new frame was adopted. The main objective of 
the Law was the vertebration of the Spanish S \& T System with a National Plan for R \& D. Priority lines were adopted, and a special emphasis was given to applied research. One of these lines was "Information and Communications Technologies" (TIC), with particular interest on Photonics and Optical Radiation Technologies. The principal instrument created in order to setup the Law was the Interministerial Commission for Science and Technology (CICYT), partly related with the Ministry of Education, but with representatives from almost every one of the ministries with $R \& D$ activities. A new instrument was in the hands of Science and Technology Policy managers and, at least during the first years of the National Plan, it provided a substantial increase in funds.

The main problems concerning Optics and related fields at that time were the lack of people, high-tech labs, and industries working in these areas. Some of the lines of the Plan were devoted to solving, if possible, these problems. An increase in doctoral scholarships, new infrastructures, and a larger amount of funds for academic and industrial projects than in previous years were the aims of the TIC programme.

But six years is not a long time to solve problems, when these problems were dated from many years back. Many of them are today still present, although some progress has been achieved. The number of $\mathrm{R} \& \mathrm{D}$ groups around Spain is growing, and a better infrastructure has been erected by some departments. But relations between industries and academic groups are still one of the main drawbacks of the system. And in the last year, because of the worldwide economic situation, the growth has not been as large as in previous years.

\section{Some Global Data}

Although some activity in Photonics are not included in official statistics, the following are the only sources available with a certain degree of accuracy. The global information we present comes from data taken from the National Plan for R \& D previously indicated. 1988 was the first year for the TIC Programme. One of the main lines was Optical Radiation Technologies and Photonics, giving special emphasis to Optical Communications and related fields. Some other areas, such as Photonics in robotics or optical sensors, were included in other national programs. Because the particular interest of our present study is Fiber and Integrated Optics, we will concentrate on the indicated Programme.

The way the National Plan operates is by the usual Call for Proposals. Once a year, normally around October, a public Call is published and every group is asked to presents its particular topic of activity. This topic has to be included in one of the broad priority lines defined by the CICYT. Because the initial objectives of the National Plan were just to activate some specific sectors, these priority lines were not too narrow. In this way, almost every one of the existing groups were a potential candidate. The number of total approved projects, from 1988 to 1991, is shown in Fig. 1. This number is, at the same time, a reflection of the total number of groups working in Spain in areas related to Optical and Photonical Technologies. A geographical distribution is shown in Fig. 2. Numbers in these two figures are somehow different. The reason is that a project can be coordinated and different regions involved in the same activity.

If it is interesting to know the number of groups, it is also important to get an approximate idea of the total number of people involved. This data are shown in Fig. 3, where the evolution with the year is indicated. 


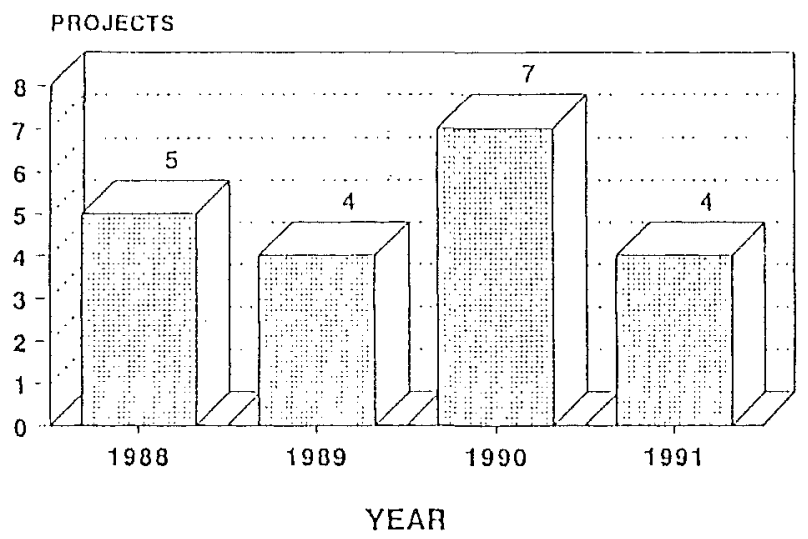

Figure 1. Number of projects (1988-1991).

\section{A Deeper Look into the Activities of the Main Groups}

As has been pointed out before, our study concerning Fiber and Integrated Optics focusses on the main groups working in these fields or closely related fields. Rather than describing each group's activity, our lines follow a thematic arrangement of topics. In this way, a certain academic or industrial group can be associated with more than one topic; according to their present interest. There is no attempt to give names; in some cases a particular researcher can be the real head of the group, but in some other cases, responsibilities are distributed, and to forget a name would create a problem! Only in some instances where the situation is very clear, a name is given. One other point needs to be clarified. The information regarding certain groups is more detailed than for others. The reason, aside from the group's activity, is that greater information here is given to groups with no paper in this special issue. We have assumed that, if a group is represented by some article, this type of information can be inferred from it.

The structure we have adopted in our study is related to the final product of the team. Namely, a broad division between Systems and Components has been taken with two main categories in the latter. The first one refers to Optical fibers and related devices (all-fiber components, couplers, and passive integrated optical components,...). The second one is related to active or semi-active components (LEDs, Diode Lasers, drivers, photodetectors,...). With respect to Systems, two different views are active in Spain now. The first one has Optical Communications as the final goal for the group activities; the second is more devoted to Sensors with specific applications, as Robotics, Domotics, or Environment.

\section{Activities in Components}

Active Components. Most of the activities in active components are derived from the general works performed, firstly, at the Telecommunication Engineering Dept (UPM) during the last seventies and, secondly, at the National Center for Microelectronics (CSIC), from the mid-eighties. Some work was initiated at the Physics Department of the UAM, but the initial group is working today more on siliconbased structures for Microelectronics, than in III-V compounds for Photonics.

At the UPM, the only group now working on this type of component is the "High-Speed Electronics Laboratory" (HISEL). The main objectives of HISEL are 


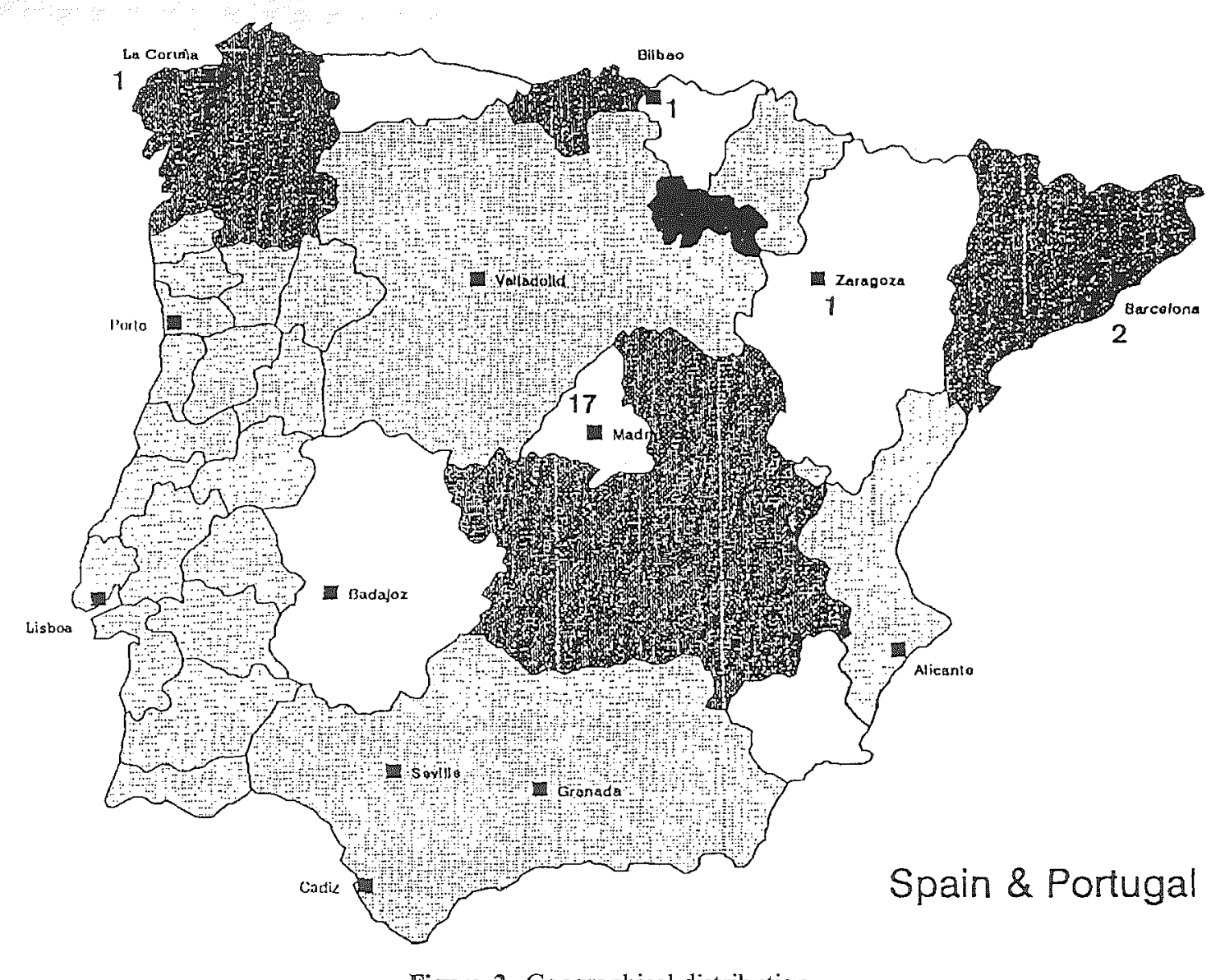

Figure 2. Geographical distribution. 


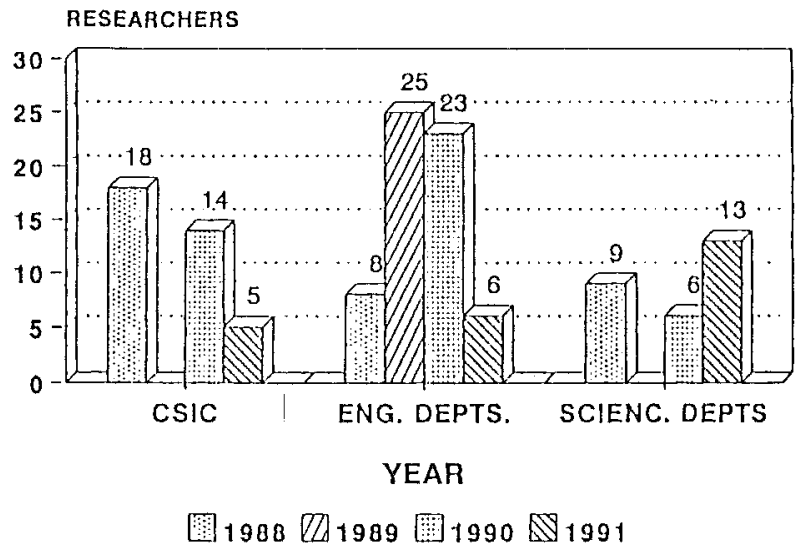

Figure 3. Number of researchers (1988-1991).

the fabrication, characterization, analysis, and simulation of electronics and communications. These devices and structures are based on III-V compounds semiconductors, and present research is focused on GaAs/GaAlAs and InGaAs/ AlInAs/InP alloy systems. Technological facilities are part of a Joint Semiconductor Laboratory, where LPE, VPE, SEM. SIMS, photolithography, UHV metallization, diffusion, annealing, and sample preparation techniques are available. A Riber $2300 \mathrm{MBE}$ system has been running since early 1990. A clean room is in operation for the MBE system. Other facilities, mainly electrical and electronic, are available too. A number of cooperative projects with European and US Universities have been carried out (Sheffield, LAAS, Cork, Stuttgart, Stanford, Boulder). Three ESPRIT projects have been the principal source of European relations.

The second active group is working at the National Centre for Microelectronics, Madrid site, of the CSIC. Its more relevant technological developments are related with studies around MQW structures and monolithic integration of optoelectronic devices on $\mathrm{Si}$ (CMOS). Although the main emphasis is toward the physical properties of devices materials and structures, an important collaboration has been maintained with some Electronics and Communications industries, both in Spain and abroad. The CEE ESPRIT Programs has been one of the places where the activity of this group has been in action. One of the present areas of interest is in integrated optical sensors, and some of the results obtained are shown in this journal.

Passive Components. According to the two above-mentioned blocks, several subdivisions could be performed here. We are going to take just two of them, although several more could appear. A final one, with some other areas, not closely related with the two previous ones, will be the last part of this group.

Integrated Optics devices. Interest on these type of devices can be traced from the beginning of the eighties, at the UPM. But no attempt to study them in depth, neither from a theoretical or practical point of view, was made until the second end of that decade. At that time, the first practical integrated optical circuit was completed, and studies of optical field propagation, by the BPM method, were achieved. Laboratory work was performed at the Microelectronics facilities of the Telecommunications Engineering Department. The first prototype, with conven- 
tional operating characteristics, was operating around 1987. Part of the team is now at the University of Cantabria, in Santander, where new facilities are being obtained. Part of the theoretical work was performed in cooperation with the IMEC, in Ghent, Belgium. This activity has been almost forgotten at the UPM.

A more active work on Integrated Optics can be seen today at several laboratories around Spain. In the Physics Department of the UAM, one of the main lines is the growing of some monocrystals, namely, $\mathrm{LiNbO}, \mathrm{Bi}_{12} \mathrm{GeO}_{20}$, $\mathrm{Bi}_{12} \mathrm{SiO}_{20}, \mathrm{Bi}_{12} \mathrm{Tio}_{20}$ y $\mathrm{CdTe}$, and optical waveguide fabrication, by proton interchange, on $\mathrm{LiNbO}_{3}$. There are several objectives of these lines, most of them of a theoretical character. Some of the present results are in this journal.

At the Universidad de Santiago de Compostela, USC, some types of integrated optical devices are nowadays being obtained, most of them by ionic diffusion. The aim of this work is an integrated collimator for use in Optical Communications. Moreover, theoretical work on nonlinear optical waveguide is being carried out at the Telecommunication Engineering Department of the UPC.

Optical fibers and all-optical fiber devices. Optical fibers, their characteristics, performances, new types, and every one of the parameters of interest for Optical Communications have been the object of study for many groups since the mid-seventies. In some cases, these studies were related to their practical implementation, both in short or long distance uses. Industries, Telefónica, and some engineering Departments were involved.

Moreover, this type of components has been one of the main objectives, during the last three years, at the Optical Communications group of the Telecommunication Engineering Department in the UPM. Filters, codifiers and decodifiers, resonators, and some other types of functions have been obtained. The present interest is also in their applications as sensors in Domotics. A special type of filter, composed by multimonomode fibers abrupt unions, has been studied, and its applications to low-cost systems considered. Main lines of research today include the use of Er-doped fibers as a part of the component and the study of nonlinear phenomena.

The University of Zaragoza, closely related with one of the two optical fiber cables industries existing in spain, has been involved for several years in this area too. Active fibers are the principal objective of its interest today.

Finally, the Daza de Valdés Institute (CSIC) was equipped three years ago, with a very complete laboratory for optical fiber measurements and characterization. It will be fully operative in the next months.

Other activities related with optical components. The study of nonlinear optical effects or components has been a main area of interest for some groups since the end of the seventies. Activity in the optical properties of liquid crystals, mainly for possible applications in Communications, appeared at the Photonics group of the UPM, in 1976. Optical bistability, Chaos, and some other nonlinear effects, mainly in liquid crystals, were published as early as in 1980. Waveguides, optical deflectors, and opto-optical modulation were obtained too. The activity decreased in the last years, but a new interest has now appeared. Some work on Optical Computing is being performed. Optical fibers, both passive and active, and nonlinear optical components are the basis of the present work. Some efforts toward the possible application of the Chaos Theory in Communications and Computing are present. Cooperative efforts with other european groups are being promoted.

The Physics Department of the UCM offers some activities in related fields too. New magneto-optic materials for optical fiber sensors are being obtained. 
Photovoltaic effects due to $\mathrm{CO}_{2}$ laser radiation on $\mathrm{Si}$ and other semiconductors are under study as they are the instabilities in pulsed lasers. These studies are more closely related with Quantum Electronics than with Integrated and Fiber Optics.

More related with our present interest are the works performed in the same department concerning scattering and propagation of electromagnetic fields in optical waveguides and fibers. Three-dimensional analysis of bending losses, coherent transfer function of hololens / parabolic optical fiber systems, and energy flux in absorbing optical waveguides are a part of the studies that have been carried out.

Moreover, and although the results are not yet definitive, holography is being employed for couplers and interconnections at UCM and UPM. Some primary results have been published in the past year.

Catalunya is the place for an intense activity in nonlinear optical effects. Two universities, UPC and UC, are involved in such studies. The objectives are Optical Communications and related fields.

\section{Activities in Systems}

Most of the work performed in this area is related to industries or private laboratories, closely connected with some industries. In this way, one of the main centers is "Telefónica I + D," a company largely dependent on Telefónica with a very impressive $R \& D$ Laboratory. Its present interest is the development of optical transmission systems with two main technology lines.

One is aimed at Intensity Modulation Direct Detection (IM-DD), and the other at Coherent Multichannel (CMC) networks. At this moment, two main projects are underway: one for obtaining line modules for the STM-4 line rate (622 $\mathrm{Mb} / \mathrm{s}$ ) for the access network, and the other for setting a CMC field trial.

Universities are not too far away from these areas. The telecommunication Engineering Department of UPC is developing a High-Performance Dense FDM Coherent Optical Network and an Optical Frequency Division Multiplexing, with high-speed FSK with Heterodyne detection. Dense Wavelength division multiplexing, with high-speed FSK, tunable filters, and direct detection is part of the present work too. In this case, because the activity is more systems than components oriented, no further details will be given here.

In Valence, at the Politechnical University, a new group has been created in the last years. Its present interest is, more theoretical than industrially oriented, but future interest is directed to establish connections with local and regional enterprises.

\section{Conclusion}

A general panorama of the spanish activity in Fiber and Integrated Optics has been presented. This view, as has been indicated previously, is more subjective than objective; it has no intention of being exhaustive. It is certain that more groups are active today and that many more will be tomorrow. But our idea has been just to offer a first glimpse. A more detailed study still needs to be done.

As a general conclusion, it is possible to indicate that the academic activity is, at this moment, at a medium level. It has improved with respect to previous years, but more efforts have to be done. In particular, relations with spanish industries are still weak and, in some cases, missing. This is a point that worries Science and Policy managers and has to be taken into account for future actions. 